\title{
An Optimized Delivery System Model in Order to Zero Ebola Cases
}

\author{
Xiaofei Cui ${ }^{1, a}$
}

${ }^{1}$ School of Electrical Engineering, North China Electric Power University, Baoding071000, China.

${ }^{\mathrm{a}} \mathrm{Cxf2015mu@163.com}$

Keywords: Immune algorithm, Graph theory, Medicine delivery system

\begin{abstract}
Based on the graph theory and immune algorithm, this paper produces a medicine delivery system in West Africa where the largest and most complex Ebola outbreak takes place. Firstly, the delivery destinations where vaccine or drug is badly needed are selected using the map of cumulative cases in different regions. Then the problem how to determine locations of distribution centers is transformed into a goal programming problem. In this model, object is defined as the minimum cost of delivery transport. The cost is calculated by the product of needed vaccine or drug in each destination and the distance from this destination to its potential delivery center. Finally, the goal programming problem is solved by immune algorithm. This model can also be used in other similar cases where such an optimized delivery system is needed urgently.
\end{abstract}

\section{Introduction}

Ebola virus disease (EVD) is a severe, often fatal illness in humanity. The most recent outbreak in West Africa has involved over 22000 individuals and caused nearly 9000 deaths [1]. Therefore, aiming to zero Ebola cases, it's important to build a model which can help to deliver medicine. In this paper, there are three steps to build and solve an optimized delivery system model:

1. Select several delivery destinations.

2. Transform the model to determine distribution centers into a goal programming problem.

3. Solve the goal programming problem by immune algorithm.

\section{Delivery Destinations}

The status with crowded confirmed cases are defined as locations of destinations that vaccine or drug delivered to. Therefore, Ebola Treatment Centers (ETC) Status may be a good choice as the compact status. Data of ETC can be found in figures from the WHO website [2]. In order to search locations of ETC which can stand for sites of regions, there are some necessary assumptions:

1. Theoretically Added Ebola Treatment Sites: In some regions, ETC haven't been built or are currently under construction. It's assumed that there are enough Ebola treatment sites.

2. Location of Added Sites: Added Ebola treatment sites mentioned in the above assumption are assumed in the center of cities. These sites can be assumed as status with crowded confirmed cases.

3. Combination of Adjacent Sites: Some regions may only have less than 50 total confirmed cases. It's assumed that no more Ebola treatment sites will be set in these locations.

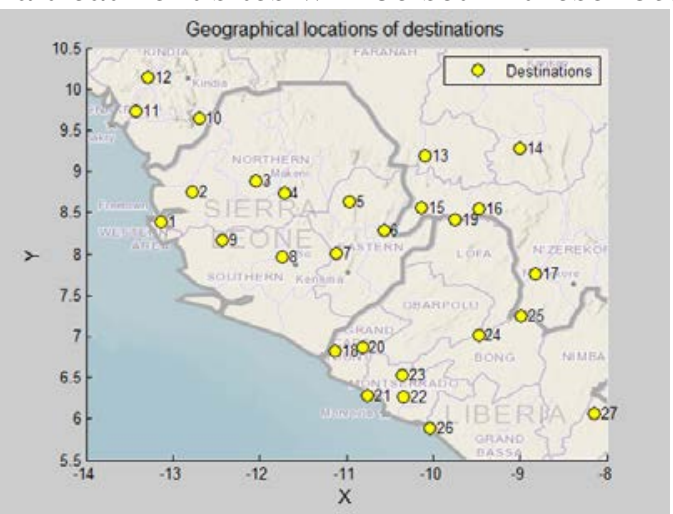

Fig.1 Geographical locations of destinations 
The result can be seen in the above figure.

In order to obtain the mathematical expression of these destinations, adjacency matrix $\mathrm{X}$ in graph theory is used in this model. At first, each element $\mathrm{X}_{\mathrm{ij}}$ is defined as the direct distance between the $\mathrm{i}$-th and the $\mathrm{j}$-th destinations. However, people cannot go from one place to another directly because of diverse obstacles, such as houses or railways. Therefore, $\mathrm{X}_{\mathrm{ij}}$ is revised considering the degree of transportation convenience.

\section{Distribution Centers}

Among destinations of delivery, to decrease costs of medication transport, how to choose distribution centers is important. The practical problem to determine locations of distribution centers can be transformed into a goal programming problem [3].

\section{Decision variable}

$\mathrm{h}_{\mathrm{j}}$ represents whether the $\mathrm{j}$-th point can be a distribution center; thus, the decision variable $\mathrm{h}_{\mathrm{j}}$ is:

$$
h_{j}=\left\{\begin{array}{lr}
1 & \text { Set the } j-\text { th point as distribution center } \\
0 & \text { Don't set the } j-\text { th point as center }
\end{array} \quad j \in M\right.
$$

where $M=\{1,2, \ldots, m\}$ stands for a set of several center alternatives.

Express $\mathrm{Z}_{\mathrm{ij}}$ in terms of the vaccine or drug of $\mathrm{i}$-th destination that is delivered by the $\mathrm{j}$-th point; so, the decision variable $\mathrm{Z}_{\mathrm{ij}}$ is:

$$
Z_{i j}=\{0,1\} \quad i \in N, j \in M
$$

whereN $=\{1,2, \ldots, \mathrm{n}\}$ stands for a set of delivery destinations.

\section{Constrains}

To confirm that supplies of a certain destination can only be delivered by one center, one constrain is defined as:

$$
\sum_{j \in M} Z_{i j}=1 \quad i \in N
$$

To avoid exceeding the maximum number of centers, the next constrain is:

$$
\sum_{j \in M} h_{j}=p \quad j \in M
$$

wherep is the maximum number of centers.

To confirm that only distribution centers can deliver supplies to other regions, the last constrain is:

\section{Object}

$$
Z_{i j} \leq h_{j} \quad i \in N, j \in M
$$

The goal of the model is to minimize the cost of delivery transport. So, the object is defined as the minimum product of needed vaccine or drug of one certain destination and the distance from this destination to its delivery center.

$$
\min F=\sum_{i \in N} \sum_{j \in M} w_{i} d_{i j} Z_{i j}
$$

wherew $_{\mathrm{i}}$ means the needed amount of vaccine and drug in the i-th destination.

Above all, the optimization model is:

$$
\begin{aligned}
& \min F=\sum_{i \in N} \sum_{j \in M} w_{i} d_{i j} Z_{i j} \\
& \text { s.t. } \quad \sum_{j \in M} Z_{i j}=1 \quad i \in N \\
& \sum_{j \in M} h_{j}=p \quad j \in M \\
& Z_{i j} \leq h_{j} \quad i \in N, j \in M \\
& Z_{i j}, h_{j}=\{0,1\} \quad i \in N, j \in M
\end{aligned}
$$




\section{Immune algorithm}

Immune algorithm belongs to the artificial immune systems field of study concerned with computational methods inspired by the process and mechanisms of the biological immune system [4]. In immune algorithm, affinity among antigens is equivalent to fitness of object, affinity among antibodies is equivalent to fitness of feasible solution, and affinity between antigen and antibody is equivalent to fitness between object and feasible solution. To avoid the premature convergence, this model relies on immune algorithm to optimize the delivery centers.

The main algorithm is described below [5].Produce initial Q antibodies randomly

$$
A b=\left\{A b_{1}, A b_{2}, \cdots, A b_{Q}\right\}
$$

Each antibody has $\mathrm{L}$ genes which is in terms of decision variables.

a. Evaluate the initial antibody swarm above. Calculate affinity between antigen and antibody.

Affinity is calculated by

$$
\operatorname{Aff}\left(A b_{i}\right)=\frac{1}{F\left(A b_{i}\right)}(i=1,2, \cdots, Q)
$$

Where $F\left(A b_{i}\right)$ means the object value of feasible solution in $A b_{i}$. $\operatorname{Aff}\left(A b_{i}\right)$ increases as $F\left(A b_{i}\right)$ decreases; thus, antibody with the highest affinity is the best antibody.

b. Clone antibodies. The number $\mathrm{N}_{\mathrm{i}}$ of clone is in proportion to affinity.

$$
N_{i}=\operatorname{Int}\left[N_{c} \frac{A f f\left(A b_{i}\right)}{\sum_{j=1}^{Q} A f f\left(A b_{j}\right)}\right](i==1,2, \cdots, Q)
$$

c. Variation of antibodies. The probability of variation is $\mathrm{P}_{\mathrm{m}}$.

d. Select better antibodies. Based on the affinity of antibodies, select antibodies with higher affinity.

e. Iterate algorithm b. to algorithm e. until antibody meet the requirement of termination.

In this model, parameters are set as table 4.

Table 1 Parameters of immune algorithm

\begin{tabular}{cc}
\hline Parameters & Value \\
\hline maximum times of iterations & 100 \\
number of initial antibodies & 50 \\
probability of variation Pm & 0.15 \\
\hline
\end{tabular}

\section{Results}

In immune algorithm, the requirement of termination is set as maximum times of iterations. The number of distribution centers is set as 6.

$\mathrm{w}_{\mathrm{i}}$ means the needed amount of vaccine and drug in the i-th destination. Since the amount of vaccine and drug is observed to be positively correlated to treatment property [6].It's considered that $\mathrm{w}_{\mathrm{i}}$ can be replaced by cumulative cases that represent the needed average degree of treatment property in each region. So the value of $\mathrm{w}_{\mathrm{i}}$ is set as cumulative cases of the $\mathrm{i}$-th destination in order to determine the best locations of distribution centers.

Process of optimization evolution can be seen in Fig.2.

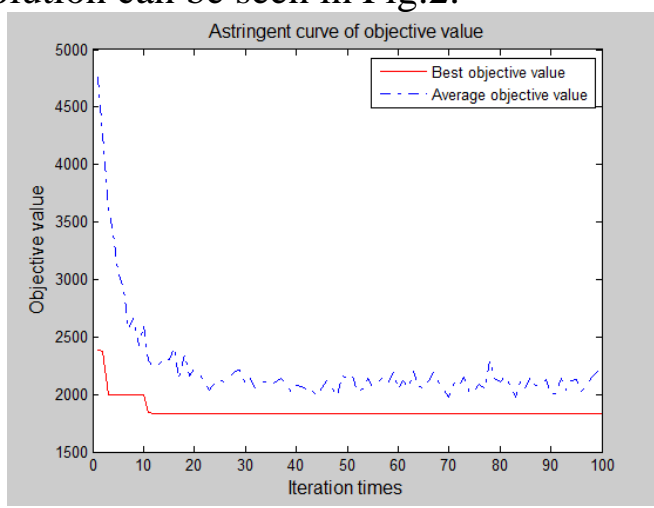

Fig.2 Astringent curve of objective value 
The best affinity is 0.000428 , while the satisfying objective value is $2.258 * 10^{3}$.

Rational distribution centers are listed in the following table.Fig. 3 shows the geographical locations of distribution centers and their destinations.

Table 2 Selection results of distribution centers

\begin{tabular}{ccccc}
\hline Distribution Centers & $\mathrm{X}$ & $\mathrm{Y}$ & Destinations & Surroundings \\
\hline 1 & -13.132 & 8.388 & 1,9 & bay and airport \\
2 & -12.775 & 8.76 & $2,10,11,12$ & bay and airport \\
3 & -12.046 & 8.883 & 3,4 & major city \\
7 & -11.116 & 8.006 & $5,6,7,8$ & airport \\
16 & -9.471 & 8.542 & $13,14,15,16,17,19,25$ & major road \\
21 & -10.761 & 6.288 & $18,20,21,22,23,24,26,27$ & bay and airport \\
\hline
\end{tabular}

The order number of the distribution centers is the same as destinations in Fig.1.

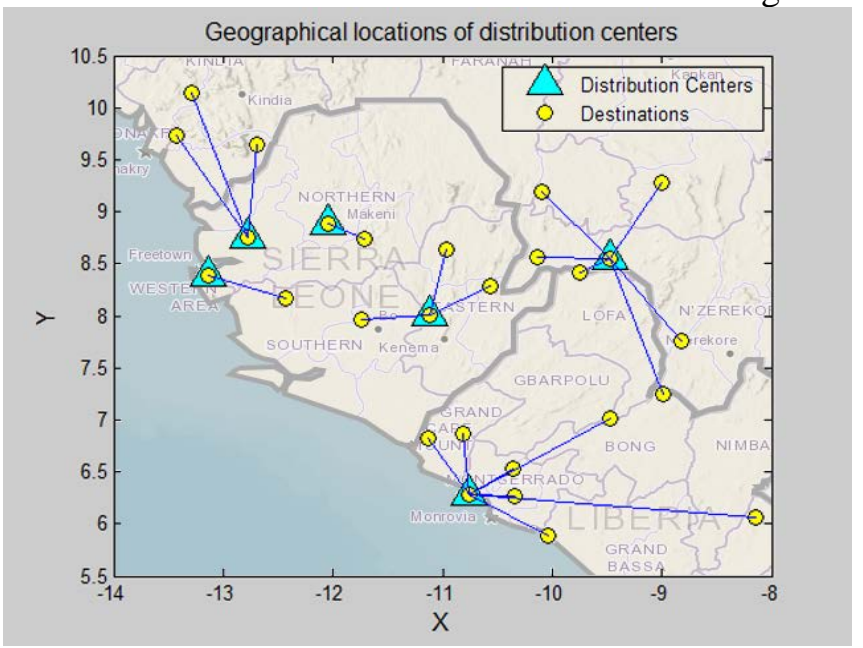

Fig.3Geographical locations of distribution centers and their destinations

Model Testing: By searching surroundings of the selected centers which can be seen in the last column of table 3, it's concluded that distribution centers are all with convenient transportation. So this model has much relation to reality.

\section{References}

[1] Information

onhttp://apps.who.int/ebola/en/ebola-situation-report/situation-reports/ebola-situation-report-4-febru ary-2015

[2] Information on http://maps.who.int/MapGallery

[3] Information on http://wenku.baidu.com/ link? U r l= r9t_z7 vix28 V w Q UH0Jo KR mg8 xn09iV B8 M8 JY S AC7 H 1Rr f D t s M C z O M Dx 5h W g C A N960w Xu7 DSP-x x K 4C h N o M o o A 36 u O Q Pf2jws6sVHHCs9cq

[4] Information on http://www.cleveralgorithms.com/nature-inspired/immune.html

[5] Mei F. Zhou, Hong T. Ye. (2012)The location of logistics distribution center based on immune algorithm. Journal of Guangxi University of Technology. 03:77-79+85.

[6] Yi Z. Li, Da N. Li, Hong Z. Guo. To investigate the relationship between aspirin dose of aspirin and salicylic acid salt and blood concentration and efficacy [J]. Clinical utility of neural disease Journal, 1994, 04: 190-193. 